\title{
Association of inflammatory biomarkers with radiological severity for COVID-19 patient risk stratification: An Indian perspective
}

\author{
Prakhar Gupta ${ }^{1}$, Ankitkumar Halani ${ }^{2}$ Tasha Samuel ${ }^{3}$, Devendra P Singh ${ }^{4}$ \\ ${ }^{1}$ Assistant Professor, ${ }^{2,3}$ PG Resident, ${ }^{4}$ Professor and Head, Department of General Medicine, L. N. Medical College \\ and J.K. Hospital, Bhopal, MP, India
}

Background: Covid - 19 caused by SARS-CoV2 was declared a global pandemic by World Health Organization. Due to rapid disease progression causing severe and fatal complications, Effective biomarkers like D - Dimer, serum ferritin, CRP, IL-6, LDH would be helpful in screening, categorization of patients, their clinical management, and prevention of serious complications, especially in areas where radiological facilities are not available immediately. Aims and Objectives: The study was aimed to investigate association between levels of inflammatory biomarkers with Covid - 19 disease severity and correlate it with HRCT chest finding to identify patients at risk of fatal complications. Materials and Methods: It was a retrospective monocentric observational study undertaken at a tertiary-care, COVID-19 dedicated centre. 200 Patients $>18$ year of age who were admitted from August 1, 2020 up to October 31,2020 with laboratory confirmed diagnosis of Covid - 19 were included in the study. Data was collected on demography, disease severity, laboratory measurements, radiology imaging retrospectively from electronic and laboratory records of patients. The disease severity was classified into mild to severe based on CT Severity scoring. HRCT Chest and inflammatory biomarkers were sent in every patient at the time of admission and the outcome was recorded. Results: There were 133 male patients, 67 female patients in our study. Average age of patients having severe lung involvement is 54.1 years, whereas Average age of patients having non-severe lung involvement is 52 years but showed No significant association with severity of lung involvement. Severity of lung involvement according to HRCT chest findings was greater in patients with raised values of both $D$ - Dimer and ferritin (RR:3.67, P value: $<0.0001)$, compared to combination of raised value of D - Dimer with LDH (RR: 2.56, P value: 0.0383 ) or D - Dimer with CRP (RR:2.22, P value:0.0157) or isolated D- Dimer (RR:2.87, $P$ value: 0.0727$)$. Individually raised levels of serum ferritin, LDH and CRP were also found to be significantly associated with radiological severity among covid-19 patients. Neither raised IL-6 levels ( $p=0.368$, RR: 0.76 ) nor did combined raised value of D - Dimer with IL-6 showed significant association with HRCT severity in our study (RR: 1.69, P value: 0.1193). Pearson correlation coefficients were also calculated and serum LDH showed strongest correlation with increasing lung involvement in HRCT chest, followed by serum ferritin. Conclusion: Based on the significant association, combination of D - Dimer with ferritin as well as isolated levels of inflammatory markers can help in assessing the severity of Covid - 19 disease based on its correlation with radiological severity, thereby it will help in immediate categorization of patients into different risk groups following diagnosis, to ensure optimal resource allocation.
Access this article online

Website:

http://nepjol.info/index.php/AJMS DOI: 10.3126/ajms.v12i4.33483

E-ISSN: 2091-0576

P-ISSN: 2467-9100

Copyright (c) 2021 Asian Journal of Medical Sciences

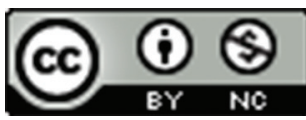

This work is licensed under a Creative Commons Attribution-NonCommercial 4.0 International License. 


\section{INTRODUCTION}

Covid - 19 caused by SARS-CoV2 was declared a global pandemic on March 11, 2020 by World Health Organization. ${ }^{1,2}$ Complexity of Covid -19 disease is centered on its unpredictable clinical course that can rapidly develop, causing severe and fatal complications.

Due to rapid disease progression, Effective biomarkers would be helpful in screening, categorization of patients, their clinical management, and prevention of serious complications. ${ }^{3}$

Biomarkers commonly evaluated to assess severity of Covid -19 diseases are D - Dimer, serum ferritin, CRP, IL-6, LDH.

D-Dimer, which is a marker of hypercoagulability, is degradation product of fibrin. Its elevated levels have been seen to be a part of the disease progression of Covid $-19 .{ }^{4}$

As per studies, CRP in severe Covid - 19patients increased significantly at the initial stage, even before CT findings\& is a signal of lung deterioration and progression. ${ }^{5}$

$\mathrm{LDH}$ (isozyme 3) present in lung tissue is expected to be released in greater amounts in the circulation with severe Covid - 19 infections, which can present as a severe form of interstitial pneumonia, often evolving into acute respiratory distress syndrome. So raised LDH can be hallmark of the severe disease. ${ }^{6}$

Activation of immune systems by this virus releases large number of cytokines, including IL-6. IL6 is a multi-effective cytokine with both anti-inflammatory and proinflammatory action $^{7}$ that contributes to host defense against infections but its excessive synthesis while fighting the virus leads to acute severe systemic inflammatory response called as cytokine storm. Role of its Plasma and/or bronchoalveolar levels as early biomarker of lung injury and as predictor of prolonged mechanical ventilation, organ dysfunctions, morbidity and mortality in lung diseases has been identified. ${ }^{8}$

Ferritin which is a degradation product of haem, has been shown to be significantly elevated in Covid- 19 patients with poorer outcomes. ${ }^{9}$

The study was aimed to investigate association between level of biomarkers with Covid-19disease severity and correlate it with HRCT chest finding to identify patients at risk of fatal complications.

\section{MATERIALS AND METHODS}

\section{Study design and patient characteristics}

It was a retrospective monocentric observational study undertaken at a tertiary-care, COVID-19 dedicated centre (L.N. Medical College and associated J.K. Hospital, Bhopal). Adult patients of more than 18 year of age who were admitted with laboratory confirmed diagnosis of Covid - 19 were included in the study. Gold-standard diagnosis of Covid - 19 is achieved through molecular identification of SARS-CoV-2 using nucleic acid amplification tests such as the reverse transcriptasequantitative polymerase chain reaction (RT-qPCR) or viral gene sequencing. ${ }^{10}$

During admission period from August 1, 2020 up to October 31,2020, total 200 patients were included, irrespective of age, gender, ethnicity or duration of symptoms of underlying illness. The study was approved by research and ethics regulatory committee of the institution. The youngest patient was 18 years old and the oldest was 87 years old.

\section{Data collection}

Data was collected retrospectively from electronic and laboratory record of patients. Confidentiality of data was ensured by hiding the medical record number of patients from data handlers. Information was collected on demography, disease severity, laboratory measurements, radiology imaging.

The disease severity was classified into mild to severe based on CT Severity scoring. The CT severity scoring was defined as sum of the individual scored in 20 lung segment regions, which may range from 0 to 40 point. Optimal inflammation load score threshold for identifying severe patients was 19.5. ${ }^{11}$

HRCT Chest and inflammatory biomarker was sent in every patient at the time of admission and outcome was logged.

\section{Statistical analysis}

Data was summarized by using descriptive statistics. P value of $<0.05$ was considered to be statistically significant. Relative risks of various markers were calculated for development of severe HRCT involvement comparing those who had elevated levels of markers and those who did not. Relative risk and odds ratio were assessed for elevated D-dimer, LDH, CRP, IL-6 and ferritin levels against normal levels. Markers were analyzed for isolated elevations as well as for various combinations and results were calculated accordingly. Average lung involvements for various groups were also calculated to assess if groups with 
elevated markers had higher mean lung involvement. ROC analysis was done wherever applicable. Pearson correlation coefficients were calculated for increasing biomarkers levels and lung involvement in HRCT chest to assess the strength of association.

\section{RESULTS}

There were 133 male patients, 66 female patients in our study. No significant association was seen between gender and severity of lung involvement ( $R R$ of $1.2, p=0.5)$.

Average age of patients having severe lung involvement is 54.1, whereas Average age of patients having non-severe lung involvement is 52 (on comparison of means, $\mathrm{p}$ value 0.421 , DF: -2.1). No significant association between age and severity of lung involvement was found.

Correlation between inflammatory markers and radiological severity shown in Figure 1.

Out of 200 patients that we have included in our study, 5 patients had isolated raised D -Dimer. It showed weak association with severe lung involvement (RR of 2.87, $\mathrm{p}=0.0862)($ Table 1).

There was a significant association seen between raised values of both $\mathrm{D}$-Dimer and Ferritin with severity of lung involvement. (RR of 3.67, p value $<0.0001$ ) (Table 1 )

We also found that 14 patients had raised values of both D -Dimer and LDH (RR of 2.56, p value - 0.0383) and 71 patients had raised values of both $\mathrm{D}-\mathrm{Dimer}$ and CRP (RR of 2.22, $\mathrm{p}$ value - 0.0157), both showed significant association (Figure 1).

The results suggest that raised D- Dimer with Ferritin showed strongest association with severity of lung involvement (based on HRCT chest) of disease when compared to combination of $\mathrm{D}$-Dimer with other inflammatory markers like LDH, CRP or isolated D Dimer.

Eighty-nine patients had raised values of both $\mathrm{D}$-dimer and IL6.The association was not significant with severity of lung involvement (RR of 1.69, p value - 0.1193) (Table 1).

In our study, there were 20 patients with raised LDH, out of which 9 were having severe lung involvement and $11 \mathrm{had}$ non-severe lung involvement. Average lung involvement in these patients is $48 \%$. In contrast, there were 180 patients with normal LDH levels, of which $32 \mathrm{had}$ severe lung involvement and $148 \mathrm{had}$ non-severe lung involvement. Average lung involvement in these patients was 30\%. (Figure 2) There was significant association between levels of $\mathrm{LDH}$ and severity of lung involvement $(p<0.0001$, RR of 2.53 , Odds Ratio of 3.78) (Table 1).

There were 76 patients with raised Ferritin, out of which 29 were having severe lung involvement and 47 had non-severe lung involvement. In contrast, there were 124 patients with normal Ferritin levels, of which 12 had severe lung involvement and 112 had nonsevere lung involvement (Figure 2). There is significant association between levels of Ferritin and severity of lung involvement $(\mathrm{p}<0.0001, \mathrm{RR}$ of 3.94 , Odds Ratio of 5.75) (Table 1).

There were 136 patients with raised IL6, out of which 26 were having severe lung involvement and $110 \mathrm{had}$ non-severe lung involvement. In contrast, there were 52 patients with normal IL6 levels, of which 13 had severe lung involvement and $39 \mathrm{had}$ non-severe lung involvement (Figure 2). There is no significant association between levels of IL6 and severity of lung involvement ( $\mathrm{p}=0.368$, RR of 0.76 , Odds Ratio of 0.70 ) (Table 1).

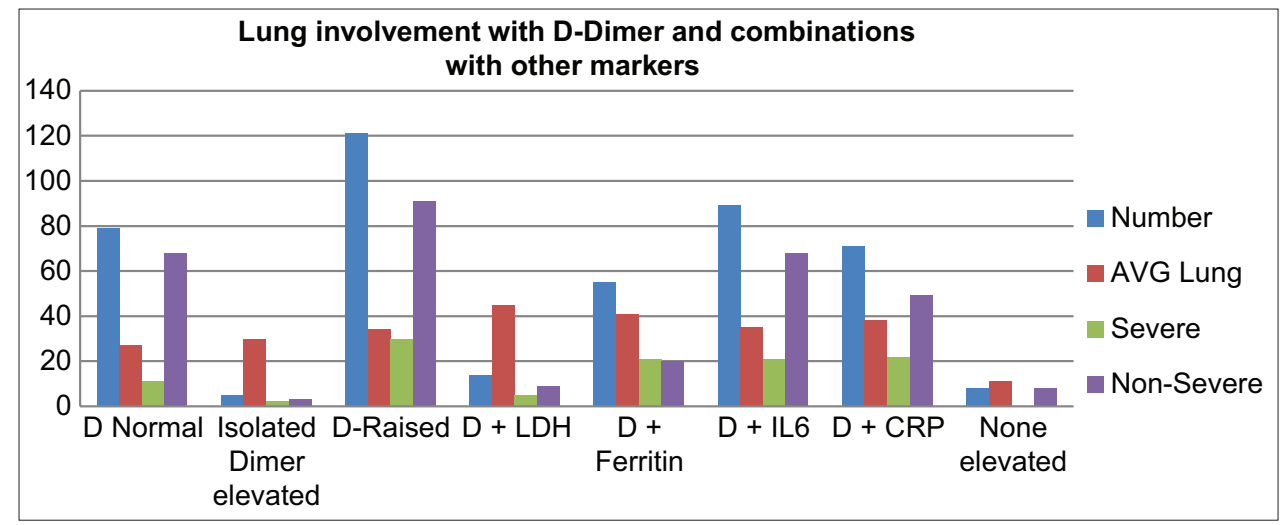

Figure 1: D: D-Dimer, LDH: Lactate dehydrogenase, CRP: C- reactive protein, IL 6: Interleukin 6 


\begin{tabular}{|c|c|c|c|c|}
\hline Biomarker & Number of Patients & $\begin{array}{c}\text { Mean Lung } \\
\text { Involvement (\%) }\end{array}$ & $\begin{array}{l}\text { Severe Lung Inv. In } \\
\text { HRCT (>50\%) }\end{array}$ & $\begin{array}{l}\text { Non-severe Lung } \\
\text { Inv. In HRCT }\end{array}$ \\
\hline None elevated & 8 & 11 & 0 & 8 \\
\hline D-dimer Normal & 79 & 27 & 11 & 68 \\
\hline D-dimer Elevated & 121 & 34 & 30 & 91 \\
\hline Isolated D-dimer raised & 5 & 30 & 2 & 3 \\
\hline D-dimer + Ferritin raised & 55 & 41 & 21 & 20 \\
\hline D-dimer + LDH raised & 14 & 45 & 5 & 9 \\
\hline D-dimer + IL-6 raised & 89 & 35 & 21 & 68 \\
\hline D-dimer + CRP raised & 71 & 38 & 22 & 49 \\
\hline LDH Raised & 20 & 48 & 9 & 11 \\
\hline LDH Normal & 180 & 30 & 32 & 148 \\
\hline Ferritin Raised & 76 & 42 & 29 & 47 \\
\hline Ferritin Normal & 124 & 25 & 12 & 112 \\
\hline IL6 Raised & 136 & 32 & 26 & 110 \\
\hline IL6 Normal & 52 & 31 & 13 & 39 \\
\hline CRP Raised & 121 & 36 & 31 & 90 \\
\hline \multirow[t]{2}{*}{ CRP Normal } & 79 & 25 & 10 & 69 \\
\hline & Relative Risk & p-value & Odds ratio & p-value \\
\hline None elevated vs & \multicolumn{4}{|c|}{ (Relative risk, Odds Ratio of developing Severe lung involvement) } \\
\hline D-dimer Elevated & 4.5 & 0.2769 & & \\
\hline Isolated D-dimer raised & 7.5 & 0.1666 & & \\
\hline D-dimer+Ferritin raised & 9.2 & 0.1082 & & \\
\hline D-dimer+LDH raised & 6.6 & 0.1825 & & \\
\hline D-dimer+IL-6 raised & 4.3 & 0.293 & & \\
\hline D-dimer+CRP raised & 5.625 & 0.2125 & & \\
\hline LDH Raised & 8.1429 & 0.1328 & & \\
\hline Ferritin Raised & 6.8961 & 0.1623 & & \\
\hline IL6 raised & 3.4818 & 0.3679 & & \\
\hline CRP Raised & 4.6475 & 0.2666 & & \\
\hline D-dimer Elevated vs Normal & 1.78 & 0.0727 & 2.038 & 0.066 \\
\hline Isolated D-dimer raised vs Normal & 2.87 & 0.0862 & 4.1212 & 0.1439 \\
\hline D-dimer+Ferritin raised vs Normal & 3.67 & $<0.0001$ & 6.4909 & $<0.0001$ \\
\hline D-dimer+LDH raised vs Normal & 2.56 & 0.0383 & 3.4343 & 0.056 \\
\hline D-dimer+IL-6 raised vs Normal & 1.69 & 0.1193 & 1.9091 & 0.1146 \\
\hline D-dimer+CRP raised vs Normal & 2.22 & 0.0157 & 2.7755 & 0.0137 \\
\hline LDH Raised vs Normal & 2.5313 & 0.0016 & 3.7841 & 0.0066 \\
\hline Ferritin Raised vs Normal & 3.943 & 0.0001 & 5.7589 & 0.0001 \\
\hline IL6 Raised vs Normal & 0.7647 & 0.368 & 0.7091 & 0.3749 \\
\hline CRP Raised vs Normal & 2.024 & 0.0346 & 2.3767 & 0.0293 \\
\hline
\end{tabular}

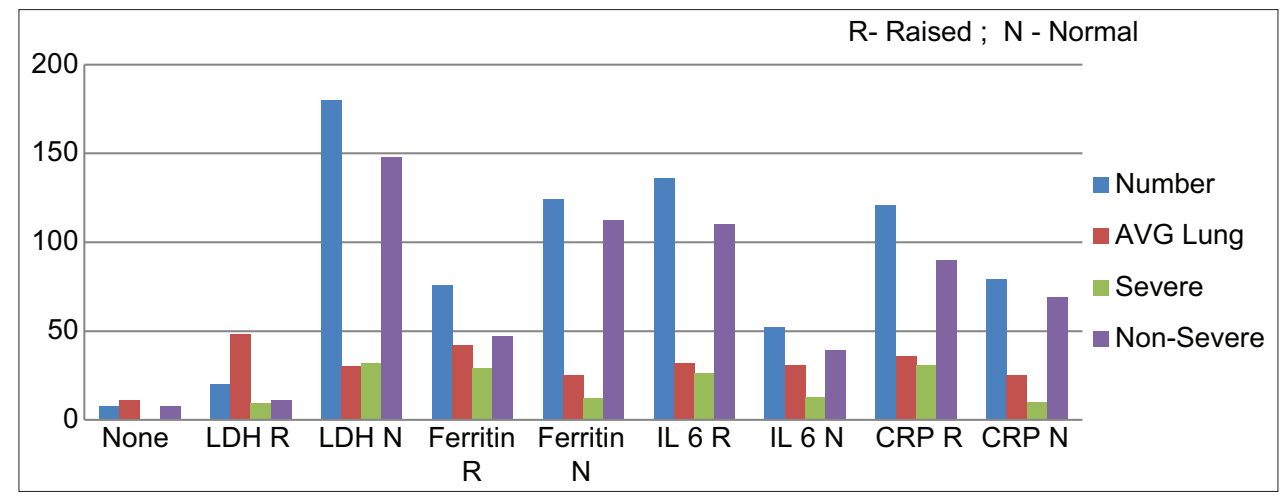

Figure 2: Significance of different inflammatory markers

There were 121 patients with raised CRP, out of which 31 were having severe lung involvement and 90 had non- severe lung involvement. In contrast, there were 79 patients with normal CRP levels, of which 10 had severe lung 
involvement and 69 had non-severe lung involvement. (Figure 2) There is significant association between levels of CRP and severity of lung involvement $(\mathrm{p}=0.0346, \mathrm{RR}$ of 2.02, Odds Ratio of 2.37) (Table 1).

We also calculated Pearson correlation coefficients of various biomarkers with increasing lung involvement in HRCT Chest (Table 2).

We performed ROC analysis for various biomarkers with respect to HRCT severity. HRCT cut-offs were taken to be $<25 \%, 25-49 \%, 50-74 \%$, = $>75 \%$ lung involvement. We found strongest association of HRCT severity with LDH followed by Ferritin, D dimer, CRP, IL6 in decreasing order (Figure 3).

\section{Table 2: Biomarkers and their respective correlation coefficients with HRCT lung involvement}

\begin{tabular}{lc} 
Biomarker & Correlation coefficient \\
\hline D-dimer & 0.096518 \\
Ferritin & 0.438796 \\
LDH & 0.511801 \\
IL-6 & 0.122706 \\
CRP & 0.103484 \\
\hline
\end{tabular}

\section{DISCUSSION}

Utility of the study is to assess the severity of Covid - 19 disease based on its correlation with level of biomarker and radiological severity.

D- Dimer and FDP were reported to be moderately/ markedly elevated in all SARS-CoV-2 deaths, suggestive of activation of coagulation processes reaching its peak in DIC. Such patients may evolve to sepsis, which is one of the most common causes of DIC. ${ }^{3}$

Ferritin is a key mediator of immune dysregulation that contributes to cytokine storm and it has been reported that fatal outcomes by Covid - 19 are accompanied by cytokine storm. Thereby those with elevated ferritin have high probability to experience serious complications. ${ }^{17}$

Our study shows stronger and significant association of raised D - Dimer with Ferritin in combination with severity of lung involvement (based on HRCT chest) of disease when compared to combination of $\mathrm{D}$-Dimer with other inflammatory markers like LDH, CRP or isolated D - Dimer.

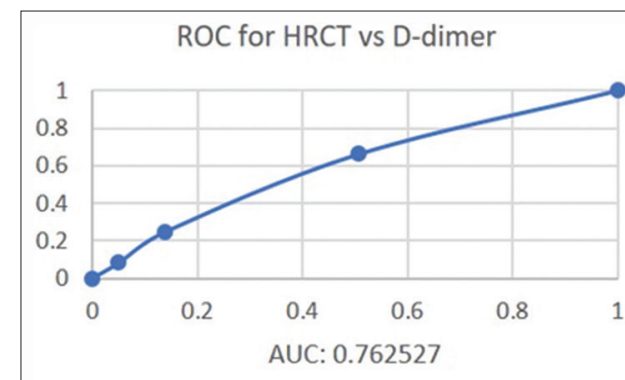

ROC for HRCT vs S. Ferritin

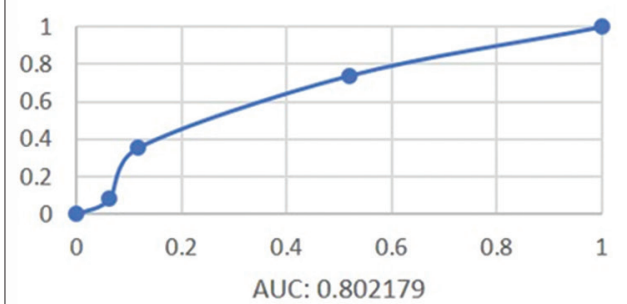

ROC for HRCT vs CRP

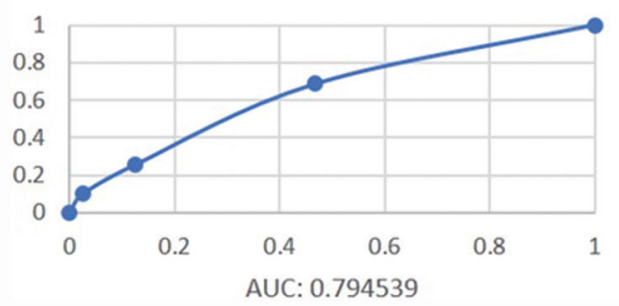

Figure 3: ROC curves for various biomarkers vs HRCT severity. HRCT cut-offs were taken to be $<25 \%, 25-49 \%, 50-74 \%$, => $75 \%$ lung involvement. (AUC: Area under curve) 
CRP is a non-specific acute-phase protein induced by IL-6 in liver and sensitive biomarker of inflammation, infection, and tissue damage. ${ }^{18}$ Studies showed that it increased significantly in severe Covid -19 patients at the initial stage, which is a signal of lung deterioration and disease progression. ${ }^{11}$ Our study confirms clinical utility of CRP levels as an indicator for severe disease and progressive inflammation. ${ }^{19} \mathrm{LDH}$, which acts as non-specific indicator of cellular death ${ }^{20}$, is present in lung tissue (isozyme 3) released in large amounts in circulation in patients with severe Covid - 19 infections present as severe form of interstitial pneumonia, often evolving into acute respiratory distress syndrome. ${ }^{17}$ There is significant association of isolated levels of LDH, CRP and ferritin with severity of lung involvement.

IL-6, which is a major pro-inflammatory mediator for induction of acute phase response its value as a prognostic biomarker in sepsis and various acute organ injuries has been extensively investigated. ${ }^{19}$

There is no significant association between levels of IL6 and severity of lung involvement in our study. There were 133 male patients, 67 female patients in our study. But age and gender showed no association with severity of lung involvement.

Based on the significant association with severity of lung involvement, Assessment of levels of various biomarkers help in immediate categorization of patients into risk groups following diagnosis, to ensure optimal resource allocation, especially in places where HRCT facilities are not readily available.

\section{ACKNOWLEDGEMENT}

The authors take this opportunity to thank Department of Radiology, Medicine and Pathology for their whole-hearted support for this study.

\section{REFERENCES}

1. WHO. Coronavirus disease (Covid - 19) outbreak. 2020. https:// www.who.int/emergencies/diseases/novel-coronavirus-2019 (accessed Apr 30, 2020).

2. Zhu N, Zhang D, Wang W, Li X, Yang B, Song J, et al. A novel coronavirus from patients with pneumonia in China, 2019. N Engl J Med. 2020; 382 (8): 727-733.

https://doi.org/10.1056/NEJMoa2001017

3. Ponti G, Maccaferri M, Cristel R, Tomasi A and Ozben T. Biomarkers associated with Covid - 19 disease progression, Critical Reviews in Clinical Laboratory Sciences. 2020; 57(6): 389-399. https://doi.org/10.1080/10408363.2020.1770685

4. Hai-Han Y, Chuan Q, Man C, Wei W and Dai-Shi T. D - Dimer level is associated with the severity of Covid -19 . Thromb Res. 2020; 195: 219-225.

https://doi.org/10.1016/j.thromres.2020.07.047

5. Tan C, Huang Y, Shi F, Tan K, Ma QT and Chen Y. C Reactive protein correlates with computed tomographic findings and predicts severe Covid - 19 early. Wiley Periodicals, Inc.J Med Virol. 2020; 92:856-862.

https://doi.org/10.1002/jmv.25871

6. Michael HB, Gaurav A, Johnny W, Stefanie B, Jens $V$ and Plebanig $M$. Lactate dehydrogenase levels predict coronavirus disease 2019(Covid- 19) severity and mortality: A pooled analysis; American Journal of Emergency Medicine. 2020; 38:1722-1726.

https://doi.org/10.1016/j.ajem.2020.05.073

7. Chi Z, Zhao W, Jia WL, Hong Z and Gui-Qiang W. Cytokine release syndrome in severe Covid- 19: interleukin-6 receptor antagonist tocilizumab may be the key to reduce mortality ; International Journal of Antimicrobial Agents. 2020; 105954.

https://doi.org/10.1016/j.ijantimicag.2020.105954

8. Huan H, Qingfeng M, Cong L, Rui L, Li Z and Wei W. Profiling serum cytokines in Covid- 19 patients reveals IL-6 and IL-10 are disease severity predictors; Emerging Microbes and Infections. 2020; 9(1): 1123-1130.

https://doi.org/10.1080/22221751.2020.1770129

9. Cooper ID, Crofts CAP, DiNicolantonio JJ, Malhotra A, Elliott B, Kyriakidou $Y$, et al. Relationships between hyperinsulinaemia, magnesium, vitamin D, thrombosis and Covid-19:rationale for clinical management. Open Heart 2020; 7: e001356.

https://doi.org/10.1136/openhrt-2020-001356

10. Laboratory testing for coronavirus disease 2019 (Covid-19) in suspected human cases: interim guidance, 2 March 2020. 2020, World Health Organization.

https://apps.who.int/iris/handle/10665/331329

11. Ran Y, Xiang L, Huan L, Yanling Z, Xianxiang Z, Qiuxia X, et al. Chest CT Severity Score: An Imaging Tool for Assessing Severe Covid - 19. Radiology: Cardiothoracic Imaging.2020; 2(2). https://doi.org/10.1148/ryct.2020200047

12. Reference Values During Pregnancy http://www.perinatology.com/ Reference/Reference\%20Range s/D-Dimer.htm) at perinatology. com. Retrieved October 2014.

13. "Ferritin" (http://www.webmd.com/a-to-z-guides/ferritin\#2). WebMD. Retrieved 27 October 2016.

14. Comel AC, Mosaner W, Bragantini D and Lanzafame M. Rapid radiological improvement of COVID-19 pneumonia after treatment with tocilizumab. Springer Nature 2020. https://doi.org/10.1007/s15010-020-01449-w

15. Herold T, Jurinovic V, Arnreich $C$, Hellmuth JC, von BergweltBaildon M, Klein M, et al. Level of IL-6 predicts respiratory failure in hospitalized symptomatic COVID-19 Patients. The Journal of Allergy and Clinical Immunology. 2020. https://doi.org/10.1016/j.jaci.2020.05.008

16. Evidence based Medicine Consult; Lab Test: C-Reactive Protein, CRP (Serum) Level; 11/22/2020; Lab Test: C-Reactive Protein, CRP (Serum) Level (ebmconsult.com)

17. Manuel V and Christian CR. Ferritin levels and Covid -19. Pan American journal of Public Health. 2020; (44): e72. https://doi.org/10.26633/RPSP.2020.72

18. Fang L, Lin L, MengDa X, Juan W, Ding L, YuSi Z, et al. Prognostic value of interleukin-6, C-reactive protein, and 
procalcitonin inpatients with Covid - 19. J Clin Virol. 2020; 127: 104370

https://doi.org/10.1016/j.jcv.2020.104370

19. Soraya GV and Ulhaq ZS. Crucial laboratory parameters in Covid-19 diagnosis and prognosis. Med Clin (Barc). 2020; 155(4): 143-151. https://doi.org/10.1016/j.medcli.2020.05.017

20. Mei-ying Wu, Yao L, Wang Y, Xin-yun Zhu, Xia-fang Wang, Pei-jun Tang, et al. Clinical evaluation of potential usefulness of serum lactate dehydrogenase (LDH) in 2019 novel coronavirus (COVID-19) pneumonia. Respir Res. 2020; 21, 171. https://doi.org/10.1186/s12931-020-01427-8

\section{Author's contribution:}

PG - Statistically analysed and interpreted, preparation of manuscript and revision of the manuscript; AH - Concept and design of the study; prepared first draft of manuscript; TS - Concept, coordination, review of literature and manuscript preparation; DPS - Interpreted the results; reviewed the literature and manuscript preparation.

\section{Work attributed to}

L. N. Medical College and J.K. Hospital, Bhopal, MP, India.

Orcid ID:

Dr. Prakhar Gupta - (1) https://orcid.org/0000-0001-9337-4244

Dr. Ankitkumar Halani - (1) https://orcid.org/0000-0003-2334-1927

Dr. Tasha Samuel - (i) https://orcid.org/0000-0003-4774-4165 